\title{
COPULATORY PLUG DOES NOT INDUCE LUTEAL ACTIVITY IN THE MOUSE MUS MUSCULUS
}

\author{
THOMAS E. McGILL, DAVID M. CORWIN* AND \\ DAVID T. HARRISON† \\ Department of Psychology, Williams College, \\ Williamstown, Massachusetts, U.S.A.
}

\section{(Received 17th Fuly 1967)}

\begin{abstract}
Summary. Female mice were mated to males that had been surgically rendered incapable of forming copulatory plugs. About half of the females were found to be pregnant, and the other half pseudopregnant, as a result of these matings. In both cases, mating with the operated males resulted in the induction of luteal activity in the female.
\end{abstract}

In the normal oestrous cycle of the female house mouse, a true luteal phase is absent. Some unknown stimulus provided by the male during mating induces the luteal phase of the oestrous cycle in the female. Land \& McGill (1967) initiated a search for this stimulus. They recognized three possible sources of stimulation from the male that might be either sufficient or necessary for the induction of luteal activity. These were: (a) a minimum number of preejaculatory thrusts, (b) the ejaculatory reflex and the formation of the copulatory plug, and (c) the subsequent presence of the plug in the vagina. Their results indicated that up to 150 pre-ejaculatory thrusts were neither sufficient nor necessary for the induction of luteal activity. On the other hand, nine of eleven animals receiving fifty-five or fewer pre-ejaculatory thrusts, plus the ejaculatory reflex and the copulatory plug, became pregnant. A third group of animals received pre-ejaculatory thrusts ranging from twenty-four to 135, plus the ejaculatory reflex and the copulatory plug which was immediately removed. Six of these females became pregnant and eight were judged pseudopregnant.

The experiment of Land and McGill failed to separate the effects of the presence of the copulatory plug in the vagina from the effects of the dramatic ejaculatory pattern exhibited by the male mouse (McGill, 1962). The present study was concerned with separating the effects of these two potential stimuli. A second purpose was to replicate and extend the findings of Land \& McGill (1967).

The method was essentially the same as that used by Land \& McGill (1967). In brief, individual, sexually-experienced males were placed in plastic cylinders. Females were then introduced into the cylinders and any resulting matings

* Present address: Department of Anthropology, Harvard University.

$\dagger$ Present address: The Medical School, Johns Hopkins University. 
were closely observed and interrupted when the female had received the appropriate treatment. Females were then caged singly for $48 \mathrm{hr}$ to ensure that the oestrous phase had passed. At this point, sexually-experienced males, called 'indicator males', were introduced into the cages of the females. Females were then examined daily for the presence of copulatory plugs produced by the indicator males, and for the birth of litters.

Females unaffected by the experimental treatment should return to oestrus in 4 or 5 days. If the experimental treatment produced pseudopregnancy, the female should not return to oestrus until 10 or 12 days following experimental treatment (Nalbandov, 1964). If the female is made pregnant by the experimental treatment a litter should be born about 19 days later.

All mice used in this experiment were of the strain $\mathrm{B} 6 \mathrm{D} 2 \mathrm{~F}_{2}$ (the second generation of a cross between inbred $\mathrm{C} 57 \mathrm{BL} / 6 \mathrm{~J}$ females and inbred $\mathrm{DBA} / 2 \mathrm{~J}$ males).

Groups studied and the results are as follows: A control group of eleven animals. These females did not receive any treatment in the plastic cylinders. They were simply placed in the home cages of indicator males and examined daily for the presence of copulatory plugs and for the birth of litters. This control group was to ensure that our females were cycling normally, that the indicator males were mating at the first opportunity, and that the resulting copulations were fertile. The eleven control females were mated within 5 days and delivered litters on schedule.

A second group consisted of ten females that received 300 pre-ejaculatory thrusts in the plastic cylinders. This represents twice the number of pre-ejaculatory thrusts experienced by the females studied by Land \& McGill (1967). Nine of these females were mated within 2 or 3 days after the introduction of the indicator males. The tenth was mated 11 days after experimental treatment.

A third group consisted of eight animals that received a normal copulation. That is, a variable number of pre-ejaculatory thrusts, plus the ejaculatory reflex and the copulatory plug, which was left undisturbed in the vagina. Seven of these animals became pregnant as a result of the treatment and one was made pseudopregnant: a fertile plug from the indicator male was found 11 days after the experimental treatment.

The results from the first three groups indicate that our sample was a fertile and sexually active one, and, in agreement with the conclusions of Land and McGill, pre-ejaculatory thrusts are neither necessary nor sufficient for the induction of luteal activity.

The fourth group studied was the critical one for the purposes of the present experiment. This group consisted of twenty females who were mated to one of four sexually experienced males that had been surgically deprived of their seminal vesicles and their coagulating glands. These males mated and ejaculated normally, but the semen did not form the typical hard, rubbery plug. The twenty females thus received a variable number of pre-ejaculatory thrusts, and the act of ejaculation without the formation of the copulatory plug. Eleven of these females became pregnant by the experimental treatment, while nine were made pseudopregnant and were mated by the indicator males on Days 10,11 or 12. Note, however, that all twenty animals had luteal activity 
induced in the absence of the formation of copulatory plugs. It is also of interest to note that all four males remained fertile. Each male 'contributed' females to both the pregnant and pseudopregnant groups. This finding leads to the hypothesis that the function of the copulatory plug in the mouse is to force spermatozoa through the cervices into the uteri and/or to aid in holding spermatozoa in the uteri.

The major conclusion from the present study is that the copulatory plug is not necessary for the induction of luteal activity in the female mouse. This result, combined with the findings of Land \& McGill (1967), narrows the search for the critical stimulus to events occurring during the ejaculatory reflex of the male mouse. Experiments are in progress to determine what mechanical, thermal, or chemical change occurs during this reflex that induces the luteal phase of the cycle.

This research was supported by Research Grant GM-07495 from the Institute of General Medical Sciences, U.S. Public Health Service.

\section{REFERENCES}

LAND, R. B. \& MaGicL, T. E. (1967) The effects of the mating pattern of the mouse on the formation of corpora lutea. 7. Reprod. Fert. 13, 121.

McGill, T. E. (1962) Sexual behavior in three inbred strains of mice. Behaviour, 19, 341.

Nalbandov, A. V. (1964) Reproductive physiology, 2nd edn, p. 285. Freeman, San Francisco. 